Monatsschrift f. Geburtshülfe u. Gynäkologie 1920;52:129-132

\title{
Gustav Klein †
}

L/as große Sterben unter den führenden deutschen Gynäkologen fordert noch immer neue Opfer.

Am 15. Juni 1920 erlag in München Gustav Klein einer schweren Erkrankung des

Blutgefäßsystems. Die ersten Anzeichen der Krankheit machten sich seit Jahr und Tag

bemerkbar, aber erst nach monatelangem hartem Ringen kam auch diese Eiche zu Fall.

Gustav Klein wurde geboren am 4. Januar 1862 in Villach in Kärnten.

Die Gymnasialzeit verbrachte er in Salzburg, die Universitätsjahre in

München und hier erwarb er 1885 die ärztliche Approbation. Die nächsten

zwei Jahre führten den jungen Arzt in verschiedene Gebiete der ärztlichen

Tätigkeit, in Würzburg und Danzig lernte Klein als ärztlicher Stellvertreter

und Assistent $\wedge^{\wedge} \gg \mathrm{MMHKiHHB}^{\wedge} \mathrm{HnB}^{\wedge \wedge \wedge} \mathrm{MHB}^{\wedge \wedge} \mathrm{B}$ Danzig und kam

die allgemeine $3 / 4^{\wedge 1} / 8^{\wedge \wedge \wedge} / 4^{7} / 8^{\wedge 3} / 4^{\wedge}{ }^{\wedge} 5 / 8 \mathrm{M}^{\wedge} \mathrm{g}^{5} / 8 \mathrm{ii}^{5} / 8^{1} / 8^{1 / 8} \mathrm{JB}$ dann als Hospi-

Praxis kennen 9 tant an die Uni-

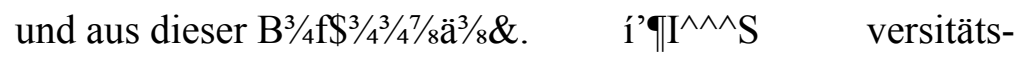

Frauenklinik in Breslau. In den Jahren 1887-89 war Klein Assistent am patho-logisch-anatomischen Institut in Breslau unter Ponfick und von dort bebielt er seine Vorliebe für anatomische und besonders histologische Forschungen. 1889 kam Klein zuHofmeiem\&ch Würzburg und Zeit bewahrte er sich sein warmes Verständnis für die Bedürfnisse und die Note des praktischen Arztes, eine Er-kenntnis, die Klein als akade-mischen Lehrer und als gericht-lichen Gutachter so wertvoll aus-zeichnete. XJber ein Jahr war er tätig als Lehrer

an der Heb-ammenschule in

ich weiß, daß er seinem Lehrer Hofmeier neben Winckel stets eine besondere Achtung und

Verehrung bewahrt hat. 1892 habilitierte er sich in Würzburg mit der Arbeit „Zur Mechanik des Ileosakralgelenkes" und kam dann noch im gleichen Jahre als Privatdozent nach München. 1901 erhielt er Titel und Rang eines außerordentlichen Universitätsprofessors. 1912 wurde er etatsmäßiger außerordentlicher Professor. Von 1901 bis zu seinem Tode war Klein Vorstand der kgl. Uníversitäts-Poliklinik (Reisingerianum).

Dieser kurze Überblick läßt erkennen, daß Klein für seine Laufbahn

keineswegs geebnete Wege und offene Türen fand; er mußte sich seinen

Weg selbst suchen und selbst bahnen, das Leben faßte ihn hart an und Klein, der lebensfrohe Genosse heiterer Stunden, der treue aufopfernde Freund, er blieb ein Alleingeher. Zu stolz, anderensein Innerstes zu erschließen, anspruchslos und hart gegen sich selbst, von angeborener Selbständigkeit Monatssohrift f. Geburtshülfe u. Gynäkologiß. Bd. LII. Heft '2. 10 130

Nachruf Gustav Klein.

und der eigenen Kraft bewußt, ging er aufrecht seinen Weg, die ruhige Sicherheit seines Gefühls und seines Urteils bewahrten ihn vor groben Irrtümern und ließen ihn die Dinge und Menschen nach ihrern wirklichen Werte einschätzen. 
In München gait es für Klein, sich eine Stellung zu schaffen. wirklich zu ,, schaffen”, d. h. aus dem Nichts hervorbringen, und das ist seinem eisernen Willen in unermüdlicher Arbeit gel·ungen. Ich habe die Zeit zum Teil miterlebt, wo Klein sich oft monatelang mit zwei bis drei Stunden Schlaf begnügte, wo ihn die aufgehende Sonne am Schreibtisch und im Labora-torium fand, und doch war er in diesen Jahren immer frisch, kannte keine Nerven, begründete seinen Ruf als akademischer Lehrer, baute sich eine glänzende Praxis alıf und sprühte von Anregung und Interesse für wissen-schaftliche Forschung. ,,Ich kenne keinen fleißigeren Menschen als Klein", sagte mir einst Chrobak. Es war eine Lust, mit einem solchen Manne zusammen zu arbeiten. Aber freilich legte er wohl damals auch schon den Grund zu seinem frühen Tode; was der Wille erzwang, das mußte der Körper büßen.

Seine augenfälligsten Erfolge feierte Klein als akademischer Lehrer; dieser Tätigkeit gait seine ganze Sorge und jede Vorlesung, jeder Kurs, jede Demonstration war aufs gründlichste vorbereitet und erschien wie aus einem Guß; Gründlichkeit, Sorgfalt, Zuverlässigkeit, waren hervor-stechende Eigenschaften Kleins. Er war ein Meister der Sprache und schrieb ein klassisches Deutsch, seit Jahren setzte er sich ein für die Verbesserung und Reinigung der deutschen Sprache, besonders in wissenschaftlichen Arbeiten. Wenn Klein in frühen Jahren in den engen Räumen an der Luisenstraße vor einer gedrängten Hörerschaft sein Kolleg ,Über die Gynäkologie des praktischen Arztes" las, dann kamen alle seine glänzenden Eigenschaften als akademischer Lehrer zur Auswirkung. Nieht zu reden von dem klaren Aufbau und der fesselnden Form des Vortrages oder von den künstlerischen Skizzen, die Kleins Meisterhand erklärend an die Tafel warf; er sprach nicht nur von Frauenkrankheiten, er sprach von der Frau, er sprach von ihr mit der Erfahrung des Arztes und mit der Begeisterung des Künstlers, er wußte historische und ïagesfragen seinem Thema ein-zuordnen und hielt seine Hörer von Anfang bis Schluß in seinem Bann.

An äußeren Schwierigkeiten gebrach es ja Kleins Lehrtätigkeit gewiß nicht, jahrelang war er genötigt, seine Kurse und Vorlesungen mit eigener Laboratoriumseinrichtung, mit eigenem Krankenmaterial, mit privaten Assistenten abzuhalten, er hielt sie ab in seinen privaten Räumen an der Luisenstraße, er mußte die ungelegensten Zeiten wählen, so am späten Mittag, und doch drängten sich die Hörer zu ihm. Diese Anhänglichkeit seiner Hörer bereitete Klein die größte Genugtuung, er liebte die aka-demische Jugend, und ihre lebhafteTeilnahme wirkte zurück auf den Lehrer, es bestand zwischen beiden ein glückliches Verhältnis gegenseitiger Anregung. Auch in den Räumen der alten Poliklinik hatte Klein noch mit den größten Widerständen der fürchteriichsten Enge und Überfüllung und der ganz unmöglichen Asepsis zu kämpfen, bis er endlich in den Pracht-räumen des Neubaues die Möglichkeit bekam, den Unterricht mit alien modernen Hilfsmitteln auszustatten.

Kleins wissenschaftliche Arbeiten waren gekennzeichnet durch Gründlichkeit und Zuverlässigkeit, Eigenschaften, die schon bedingt werden

NacL·ruf Gustav Klein.

131

durch den großen Umfang, welchen nüchterne anatomische Untersuchungen in seinen Arbeiten einnehmen. In München fehlte ihm zunächst alles klinische Material, er richtete sich selbst ein Laboratorium ein, und als Früchte jener Zeit erschienen zahlreiche Mitteilungen von ihm und seinen Schülern zur Anatomie und Entwicklungsgesehichte der weiblichen Genital -organe. Aus diesen Forschungen erwuchsen breit angelegte Untersuchungen über vergleichende Anatomie und Entwicklungsgesehichte, welche Klein gemeinsam mit dem genialen Forscher Karl Groschuff durchführte. Klein. widmete diesen Arbeiten jahrelang seine Nächte und seine Ferien; 
durch die Ungunst der Umstände und besonders durch Groschu^fs allzufrühen Tod blieben die Arbeiten unvollendet und die bereits vorliegenden Ergebnisse wurden nie veröffentlicht. Zahlreich sind Kleins klinische Arbeiten, und auch allgemeinen und ferner liegenden Fragen brachte er lebhaftes Interesse entgegen: Narkose und Anästhesie, Blutuntersuchungen, Hebammenwesen, Mittelschul-vorbildung der Mediziner, Lander ziehungsheime, Bevölkerungsfragen. In der Frage des Frauenstudiums nahm er frühzeitig eine selbständige Stellung ein; in einer Zeit, wo der Frau die medizinischen Hörsäle noch streng verschlossen waren, bekannte sich Klein frei und offen zu dem Grund-satze der Gleichberechtigung der Frau im Medizinstudium. Mit aller Energie beteiligte sich Klein am Kampfe gegen den Gebärmutterkrebs, dieser Aufgabe gal ten zahlreiche Arbeiten über Operation und Operations erfolge in der Karzinom-Therapie und in warmen Worten wandte er sich an die praktischen Ärzte um Unterstützung im Kampfe gegen die Geißel des Karzinoms. Als dann in der StrahlenTherapie eine verheißungsvolle Waffe gegen den Krebs winkte, da war Klein der erste, welcher seinen Klientinnen alle Vorteile dieses Verfahrens zuzuwenden bestrebt war. In zähem Ringen und mit überzeugenden Darlegungen überwand er die Wider -stände, welche der Poliklinik diese Hilfsmittel vorenthalten wollten, und in alter Gründlichkeit machte er sich mit den theoretischen und praktischen Grundlagen des Verfahrens vertraut und las in dankenswerter Weise selbst ein $\mathrm{f}$ esselndes Kolleg für praktischeÄrzte über die physikalischen Grundlagen der Strahlen-Therapie. In all den Jahren arbeitete er mit seinen Schülern intensiv mit an der Weiterentwicklung und Verbesserung der Strahlen-Therapie und zahlreiche Arbeiten berichten über die Fortschritte und Erfolge des Verfahrens an der Poliklinik und in der Privatpraxis Kleins.

Aufs regste beteiligte sich Klein am wissenschaftlichen Vereinsleben; auf den Tagungen der deutschen Gesellschaft für Gynäkologie war seine hohe markante Gestalt eine regelmäßige und von vielen Fachgenossen herzlich begrüßte Erscheinung. Er durfte es sich erlauben, in Kongreßberichten die Unsitte zu rügen, daß auf diesen Tagungen einzelne Vorträge mit lautem Beifall ausgezeichnet wurden, denn er selbst war unter den, also Ausgezeichneten.

Als Arzt war Klein, entsprechend seinem ganzen Wesen, von unüber-troffener

Gewissenhaftigkeit, die Indikationsstellung zu einer Operation geschah mit der größten Sorgfalt, und die Nur-Techniker, das indikations-lose Operieren bekämpfte er in Wort und Schrift und stand nicht an, solches Verfahren aufs schärfste zu brandmarken. Der suggestive Einfluß, der von der Person eines großen Arztes ausgeht und den inehr oder weniger jeder Arzt besitzen muß, er war Klein in ganz außergewöhnlichem Maße

$10 *$

132

Nachruf Gustav Klein.

eigen. Die ruhige Sicherheit, die Bestimmtheit und Güte wirkten auf den Hilfesuchenden und gaben ihm das Gefühl, geborgen zu sein. Klein hatte ein offenes Herz fur alles menschliche Unglück; daß er auch eine stets offene Hand hatte, das wissen nur die wenigsten. Güte und Menschen-liebe war séin innerstes Wesen, aber sein Wohltun betätigte sich nicht auf offenem Markte. Bezeichnend ist, daß eine hiesige Arbeiter-Zeitung seiner in einem Nachrufe als des Wohltäters der arroen Frauen gedachte.

Klein hatte eine glückliche Gabe, auch unter schwierigen äußeren Verhältnissen begeisterte Mitarbeiter zu gewinnen; an dem Ergehen seiner Schüler nahm er wärmsten persönlichen Anteil und er förderte sie noch nach Jahren in der selbstlosesten Weise, wo es ihm nur möglich war; gerne feierte er mit ihnen frohen Semesterschluß. Einblick in sein innerstes Wesen gewannen aber auch von seinen nächsten Mitarbeitern nur wenige, und das ist wohl der Grund, warum sich 
die freundlichen Beziehungen nur selten zu richtiger Freundschaft entwickelten. Klein selbst gab dem noch in seinen letzten Tagen Ausdruck mit den Worten: , Ich bin oft verkannt worden”. Kleins Lebensbild bleibt unvollständig und unverstanden, wenn man über dem Lehrer, Forscher und Arzt den Künstler vergißt; Klein war Künstler nicht nur als Kunstfreund und Kunstkenner, sondern nach seiner ganzen Lebensauffassung, und der künstlerische Einschlag war die besondere Note in seinem Wesen. Sein Blick war nicht gefangen durch seine Fachwissenschaft oder gar durch Einzelfragen, er schaute weiter, und philosophische und religiose Fragen im weitesten Sinne beschäftigten ihn. Daher kam es, daß ihn die Naturwissenschaften, besonders die Ent-wicklungsgeschichte, so sehr anzogen, daher stammt seine Neigung, positive Erkenntnisse spekulativ zu verwerten, und das ist auch der tiefere Grund, der ihn letzten Endes zum Studium der Geschichte der Medizin und zu den alten Meistern fiihrte. Seine historischen Studien haben Klein in den weitesten Kreisen nicht nur der Fachgenossen bekanntgemacht. Aus kleinen Anfängen brachte er eine selten reiche Sammlung alter Original-werke zusammen, die mit dem feinen Verständnisse des Kenners und Künstlers ausgewählt waren; die Liebe und Verehrung aber, die er den alten Meistern entgegenbrachte, die lohnten sie ihm wieder, ihm redeten sie eine verständliche Sprache, ihm eröffneten sie ihre geheimen Schätze, und er geizte nicht, seine reichen Funde der Allgemeinheit in Wort und Schrift iind Bild zugänglich zu machen.

Begeistert für alles Hohe und Edle nahm dieser Mann mit ganzer Seele teil an dem Schicksale des Vaterlandes, an seinem stolzen Glanze, an seiner tiefen Trauer. Vom Anfange des Krieges an war er in einem Heimatlazarett angestrengt tätig, dann litt ,, es ihn nicht mehr herinnen”, er meldete sich freiwillig ins Feld. Mit gerechtem Stolze trug er sein wohlverdientes eisernes Kreuz.

Es ist schwer, sich dem harten Schicksale zu beugen, das einen solchen Mann des Wollens und des Könnens aus der Vollkraft seines Schaffens herausreißt; er selbst hatte noch bis in die letzte Zeít große Arbeitspläne.

Klein hat seine Jahre genutzt und hat mit seinen Talenten gewuchert. Seinen Schülern bleibt sein Beispiel als edelstes Vermächtnis.

Anton Hengge.

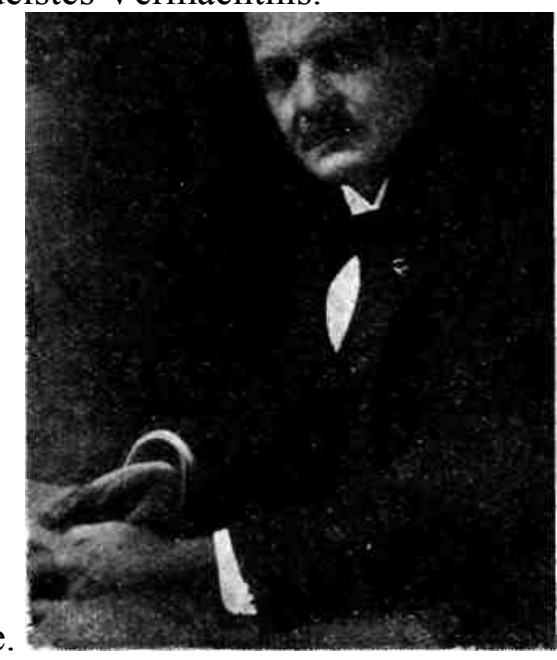

